\title{
Crack Detection with Lamb Wave Wavenumber Analysis
}

\author{
Zhenhua Tian ${ }^{1 *}$, Cara Leckey ${ }^{2}$, Matt Rogge ${ }^{2}$, Lingyu $\mathrm{Yu}^{1}$ \\ ${ }^{1}$ Mechanical Engineering Department, University of South Carolina, Columbia, SC \\ ${ }^{2}$ NASA Langley Research Center, Nondestructive Evaluation Science Branch, Hampton VA
}

\begin{abstract}
In this work, we present our study of Lamb wave crack detection using wavenumber analysis. The aim is to demonstrate the application of wavenumber analysis to 3D Lamb wave data to enable damage detection. The 3D wavefields (including $v_{x}, v_{y}$ and $v_{z}$ components) in time-space domain contain a wealth of information regarding the propagating waves in a damaged plate. For crack detection, three wavenumber analysis techniques are used: (i) two dimensional Fourier transform (2D-FT) which can transform the time-space wavefield into frequency-wavenumber representation while losing the spatial information; (ii) short space 2D-FT which can obtain the frequency-wavenumber spectra at various spatial locations, resulting in a space-frequency-wavenumber representation; (iii) local wavenumber analysis which can provide the distribution of the effective wavenumbers at different locations. All of these concepts are demonstrated through a numerical simulation example of an aluminum plate with a crack. The 3D elastodynamic finite integration technique (EFIT) was used to obtain the 3D wavefields, of which the $v_{z}$ (out-of-plane) wave component is compared with the experimental measurement obtained from a scanning laser Doppler vibrometer (SLDV) for verification purposes. The experimental and simulated results are found to be in close agreement. The application of wavenumber analysis on 3D EFIT simulation data shows the effectiveness of the analysis for crack detection.
\end{abstract}

Keywords: : Lamb wave, crack detection, wavenumber analysis, EFIT modeling

\section{INTRODUCTION}

Lamb waves are a subset of guided waves that propagate between two parallel surfaces, such as shell and plate structures [1]. They have proven to be useful for structural health monitoring (SHM) and damage detection in plate-like structures due to their attractive features including the capability of travelling long distances with less energy loss and sensitivity to small defects in the structure [2-6]. The fundamentals of this type of damage detection consist of evaluating the characteristics of wave propagation along the wave path between wave actuator and receiver. Many researchers have contributed to the study of SHM using Lamb wave propagation methods [3-6]. Although recent advances in Lamb wave based SHM technology have demonstrated the feasibility of detecting and locating damage in structural components, there remains many challenging problems for real-world implementation due to the complexity involved with propagating Lamb waves.

Lamb waves are known for being multimodal. From the Rayleigh-Lamb equation, the relationship between frequency $f$ and wavenumber $k$ can be found. Once the $f-k$ relations are known, group and phase velocity dispersion curves of different modes can be calculated [2]. Figure 1 gives examples of dispersion curves of a 1-mm thick 2024-T3 aluminum plate (material properties are listed in Table 1). The dispersion curves show that at the frequency-thickness values lower than $1.5 \mathrm{MHz} \cdot \mathrm{mm}$, only the fundamental $\mathrm{A}_{0}$ and $\mathrm{S}_{0}$ Lamb modes exist. If the thickness is held as a constant, then as the frequency increases, more Lamb wave modes (such as $\mathrm{A}_{1}, \mathrm{~A}_{2}, \mathrm{~S}_{1}$ and $\mathrm{S}_{2}$ ) appear. For many SHM applications, frequencies are usually chosen below a critical value where only $\mathrm{A}_{0}$ and $\mathrm{S}_{0}$ modes exist in order to keep the wave propagation patterns and pertinent data analysis simple. However, even when a single mode is actuated for inspection, various other modes may still be generated via mode conversion due to interaction with structural features such as boundaries, notches, stiffeners, and thickness changes.

Various transducers have been utilized to actuate or acquire Lamb waves. One commonly used Lamb wave actuator is the thin wafer piezoelectric sensor (referred to as PZT in the rest of the paper). Intensive research activities have been conducted on this sensor type. A detailed description of the use of PZT sensors can be found in [7]. Recently, the

${ }^{*}$ Corresponding author. Email: tianz@email.sc.edu 
scanning laser Doppler vibrometers (SLDV) have been used as a means for non-contact remote sensing in ultrasonic wavefield measurement due to the fact that these systems can make accurate surface velocity/displacement measurements over a spatially-dense grid and provide high resolution image sequences of wave propagation [8-20]. Specifically, Staszewski et al. illustrated the concept of Lamb wave sensing utilizing a 1D laser Doppler vibrometer [12]. Swenson et al. compared 1D and 3D SLDV measurements in the time domain for Lamb waves that were excited by a PZT [8]. Olson et al. used 1D and 3D SLDV to provide Lamb wave finite element modeling validation via time domain comparisons [17]. Rogge and Johnston investigated the use of the continuous wavelet transform for defect sizing and characterization in wavefields measured in composite rods and metallic plates [20]. Ruzzene [14] and Michaels et al. [15, 16] measured full wavefields with a 1D scanning laser Doppler vibrometer. The resulting wavefield data was analyzed with a frequency-wavenumber approach for separating incident and reflected waves.

The increase in availability of large computational resources, such as computing clusters, has made realistic threedimensional (3D) elastic wave simulation more feasible. Similar to the experimental wavefields recorded by 3D SLDV techniques, 3D elastic wave simulations also yield the 3D data. However, such simulations yield not only wavefield data for the surface of a simulated specimen, but also throughout the sample thickness. Simulation tools are expected to play a key role in the development of practical, cost-effective SHM systems since it is not feasible to rely only on experimental techniques for SHM system optimization and validation. For example, optimizing Lamb wave based SHM systems will likely require thoroughly investigating variations on sensor number and sensor placement with respect to a very large number of expected damage types, sizes, shapes, and locations. In fact, model-assisted SHM validation is likely the most practical, and perhaps the only viable approach for establishing confidence in the damage detection capabilities of SHM systems. These factors led us to investigate how simulation can aid the development of Lamb wave SHM systems. In this work, the 3D elastodynamic finite integration technique (EFIT) was strategically chosen as the simulation method for reasons described in previous publications [21, 22].

In this paper, we present our study of 3D Lamb wave propagation characterization and crack detection using wavenumber analysis. The 3D Lamb wave data (including velocity components $v_{x}, v_{y}$ and $v_{z}$ ) in time-space domain contain a wealth of information regarding the wave propagation. Three wavenumber analysis techniques are used: (i) using two dimensional Fourier transform, the wavefield in time-space domain is transformed to the frequencywavenumber domain, where various Lamb wave modes appear discernible. (ii) to retain the spatial information that is lost during the Fourier transformation, a short space Fourier transform is adopted to obtain the frequency-wavenumber spectra at various spatial locations in 1D, resulting in a space-frequency-wavenumber representation (iii) local wavenumber analysis is used to acquire the effective wavenumber distribution in the $\mathrm{x}-\mathrm{y} 2 \mathrm{D}$ spatial domain for crack detection. All three methods are described in detail in section 2. The EFIT simulation is introduced in section 3, and is used to simulate Lamb wave propagation in an aluminum plate. The EFIT out-of-plane results are experimentally verified through comparisons with $1 \mathrm{D}$ SLDV measurements. The wavenumber analysis techniques are applied to the 3D EFIT simulation data for crack detection in section 4.

Table 1 Material properties

\begin{tabular}{ll}
\hline Material properties & Aluminum-2024-T3 \\
\hline Density $\left(\mathrm{kg} / \mathrm{m}^{3}\right)$ & 2780 \\
Young's modulus $(\mathrm{GPa})$ & 72.4 \\
Poisson's ratio & 0.33 \\
\hline
\end{tabular}

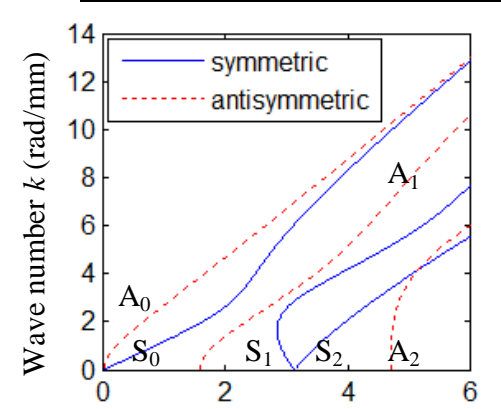

(a)

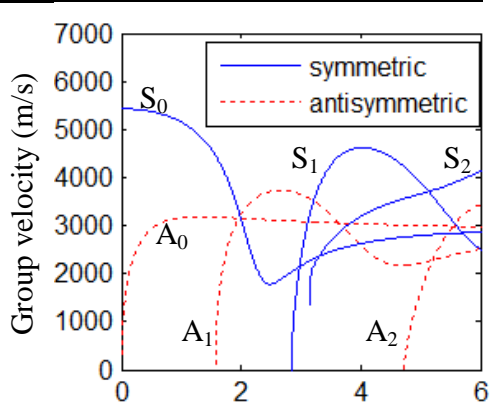

(b)

Figure 1 Dispersion curves of a 1-mm thick 2024-T3 aluminum plate: (a) frequency-wavenumber dispersion curves, (b) group velocity dispersion curves. 


\section{WAVENUMBER ANALYSIS}

For crack detection, three wavenumber analysis techniques are discussed: (i) frequency-wavenumber analysis; (ii) space frequency-wavenumber analysis; (iii) 2D local wavenumber analysis.

\subsection{Frequency-wavenumber analysis}

The Fourier transform has proved to be an enormously useful tool for analyzing time series. The concept of Fourier analysis is straightforwardly extended to multidimensional signals such as the time-space full wavefield data $u(t, \mathbf{x})$, which is in terms of time variable $t$ and the space vector $\mathbf{x}$ [23]. The time-space Fourier transform can be written as,

$$
U(\omega, \mathbf{k})=\int_{-\infty}^{\infty} \int_{-\infty}^{\infty} u(t, \mathbf{x}) e^{-j(\omega t-\mathbf{k} \cdot \mathbf{x})} d t d \mathbf{x}
$$

where the wavenumber vector $\mathbf{k}$ and space vector $\mathbf{x}$ are

$$
\mathbf{k}=\left(k_{x}, k_{y}, k_{z}\right) \text { and } \mathbf{x}=(x, y, z)
$$

The frequency-wavenumber representation $U(\omega, \mathbf{k})$ can be interpreted as an alternative representation of the wavefield $u(t, \mathbf{x})$ in terms of the temporal frequency variable $\omega$ and the wavenumber vector $\mathbf{k}$. The fundamental base of the timespace Fourier transform is a harmonic function $e^{j(\omega t-\mathbf{k} \cdot \mathbf{x})}$. In particular, for the study of wave propagation in a certain direction $x$, the full wavefield $u(t, \mathbf{x})$ reduces to the wavefield $u(t, x)$, which is in terms of the time variable $t$ and the space variable $x$. Accordingly, the time-space Fourier transform in Eq. (1) reduces to a 2D FT, which can be expressed as [23]

$$
U\left(\omega, k_{x}\right)=\mathrm{F}_{2 \mathrm{D}}[u(t, x)]=\int_{-\infty}^{\infty} \int_{-\infty}^{\infty} u(t, x) e^{-j\left(\omega t-k_{x} x\right)} d t d x
$$

where $\mathrm{F}_{2 \mathrm{D}}$ is the $2 \mathrm{D}$ FT process that can transform $u(t, x)$ to the representation $U(\omega, k)$ in the frequency-wavenumber domain.

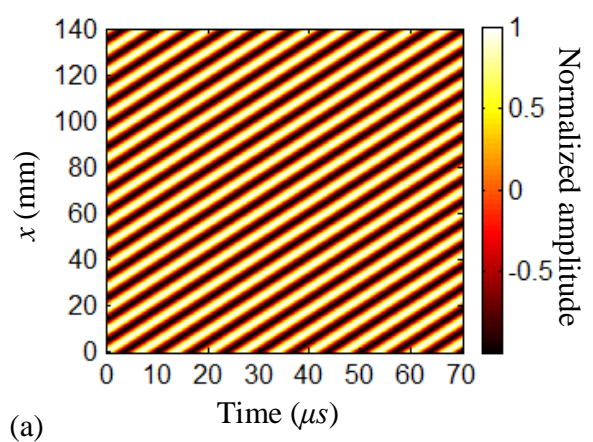

(a)

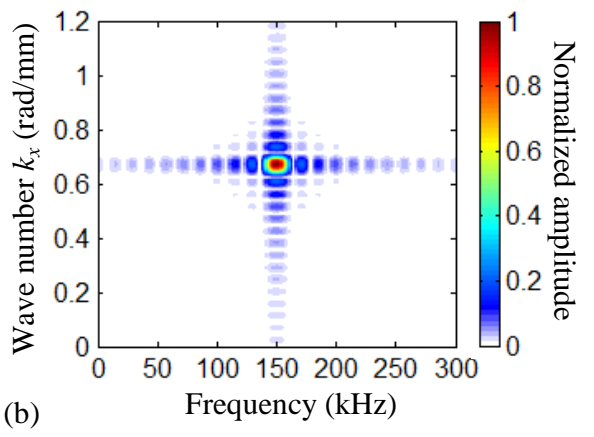

(b)

Figure 2 2D FT of a single frequency single mode numerical wavefield $u(t, x):$ (a) time-space wavefield; (b) frequencywavenumber spectrum.

Figure 2 gives an example of frequency-wavenumber analysis of a single frequency single mode numerical wavefield $u(t, x)$. Figure $2 \mathrm{a}$ is the time-space wavefield $u(t, x)$ in terms of amplitude (color map) versus time and spatial location. Figure $2 b$ gives the frequency-wavenumber spectrum in terms of amplitude (color map) versus frequency and wavenumber. The spectrum has the maximum value at $(150 \mathrm{kHz}, 0.68 \mathrm{rad} / \mathrm{mm}$ ), indicating the harmonic (frequency, wavenumber) component. Note that the small ripples around the spectrum center $(150 \mathrm{kHz}, 0.68 \mathrm{rad} / \mathrm{mm}) \mathrm{are}$ caused by Fourier transform leakage.

\subsection{Space-frequency-wavenumber analysis}

The 2D FT of the wavefield data $u(t, x)$ to the frequency-wavenumber domain unveils wave propagation characteristics that cannot be explicitly seen in the time-space domain. However, this approach cannot retain the space or time information. It will be beneficial if the relationship between the location and the frequency/wavenumber can be maintained. 
Similar to the idea of the short time Fourier transform using a windowing technique [24], a short space 2D FT is adopted in this paper to perform the space-frequency-wavenumber analysis where the spatial information is retained. The idea is a straightforward extension of short time Fourier transform to the two dimensional problem, breaking down the timespace wavefield into small segments over the space dimension before Fourier transformation. To do this, the wavefield data is multiplied by a 2D window function which is non-zero for only a short period of space while constant over the entire time dimension. The 2D FT of the resulting wavefield segment is then taken as the window sliding along the space axis. By this means, frequency-wavenumber spectra at various spatial locations are obtained, resulting in a threedimensional representation of the signal, referred to as the short space 2D FT. Mathematically, this process is written as:

$$
Z\left(\alpha, \omega, k_{x}\right)=\int_{-\infty}^{\infty} \int_{-\infty}^{\infty} u(t, x) W^{*}(t, x-\alpha) e^{-j\left(\omega t-k_{x} x\right)} d t d x
$$

where $\alpha$ is the space index and $W(t, x)$ is the $2 \mathrm{D}$ window function. Any commonly used window function such as Hanning or Guassian can be adapted for the 2D case. In our study, a Hanning function is used to construct the 2D window $W(t, x)$, giving:

$$
W(t, x)=\left\{\begin{array}{cc}
0.5\left[1-\cos \left(2 \pi \frac{x}{D_{x}}\right)\right] & \text { if }|x| \leq D_{x} / 2 \\
0 & \text { otherwise }
\end{array}\right.
$$

where $D_{x}$ is the window length in the space domain. Note that during the short space 2D FT operation, at each position $\alpha$, the window needs to overlap with the previous position to reduce artifacts at the boundary. The Fourier transform of the windowed portion will yield a frequency-wavenumber spectrum centered around the position $\alpha$. The resulting short space 2D FT indicates how the frequency-wavenumber spectra vary in space.

Figure 3 shows an example of the short space FT for the the single frequency single mode time-space wavefield $u(t, x)$ in Figure 2a. An illustration of the moving window is shown in Figure 3a. By sliding the window along the space dimension and stacking the corresponding frequency-wavenumber spectra, a space-frequency-wavenumber spectrum is obtained. Figure $3 \mathrm{~b}$ plots the resulted space-wavenumber slide at a selected frequency $150 \mathrm{kHz}$, showing how wavenumber changes along the propagation distance, staying constant for the single frequency single mode single.

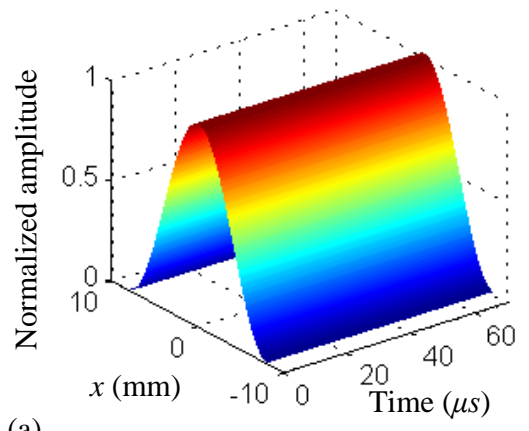

(a)

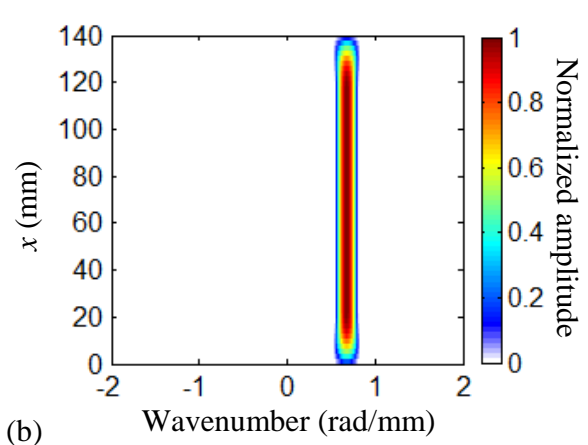

Figure 3 Short space 2D FT of a single frequency single mode numerical wavefield $u(t, x):$ (a) time-space window; (b) space-wavenumber spectrum at the frequency $150 \mathrm{kHz}$.

\subsection{Local wavenumber domain analysis}

For the study of wave propagation in $2 \mathrm{D} x-y$ space domain, the full wavefield $u(t, \mathbf{x})$ in Eq. (1) is reducedto the wavefield $u(t, x, y)$, which is in terms of the time variable $t$ and the space variables $x$ and $y$. Accordingly, the timespace Fourier transform in Eq. (1) changes to a 3D FT, which can be expressed as [23]

$$
U\left(\omega, k_{x}, k_{y}\right)=\mathrm{F}_{3 \mathrm{D}}[u(t, x, y)]=\int_{-\infty}^{\infty} \int_{-\infty}^{\infty} \int_{-\infty}^{\infty} u(t, x, y) e^{-j\left(\omega t-k_{x} x-k_{y} y\right)} d t d x d y
$$

where $\mathrm{F}_{3 \mathrm{D}}$ is the $3 \mathrm{D}$ FT process that can transform $u(t, x, y)$ to the representation $U\left(\omega, k_{x}, k_{y}\right)$ in the frequencywavenumber domain. Figure 4 shows an example of 3D FT of the wave propagation in 2D space domain. The wavefield 
$u(t, x, y)$ is plotted as amplitude (in color) versus time $t$ and spatial dimensions $x$ and $y$. The frequency wavenumber spectrum is plotted as amplitude (in color) versus frequency $f$ and wavenumbers $k_{x}$ and $k_{y}$. However, once the 3D FT is performed, the space information is lost and it is difficult to relate features in the complex spectrum to properties of localized damage. The goal of the local wavenumber analysis method is to maintain the spatial dependency while providing wavenumber domain information.

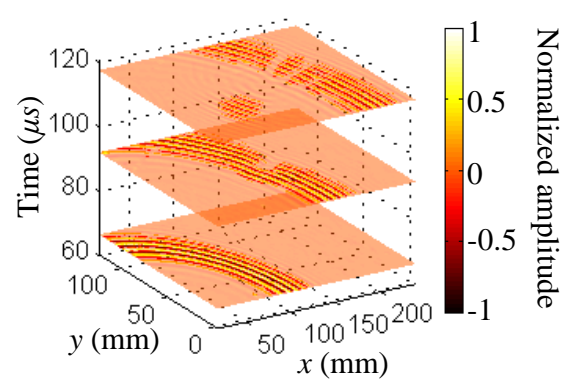

(a)

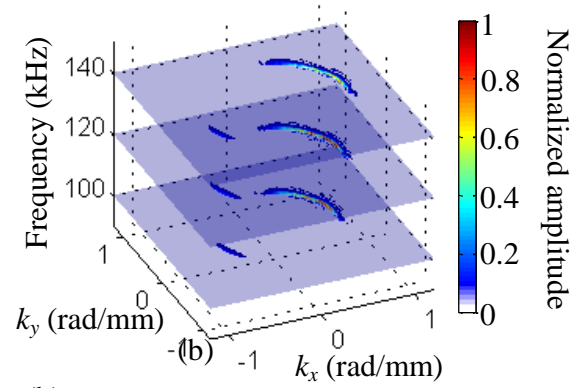

(b)

Figure 4 3D FT of the wave propagation in 2D space domain: (a) time-space wavefield for the wave propagation in 2D space domain; (b) frequency-wavenumber spectrum.

The local wavenumber domain analysis is accomplished by implementing a 3D windowed Fourier Transform where the wavefield is windowed in spatial dimensions (both $\mathrm{x}$ and $\mathrm{y}$ dimensions) and the 3D FT is performed to produce the frequency-wavenumber domain representation [25]. Mathematically, the process can be written as:

$$
L\left(\alpha, \beta, \omega, k_{x}, k_{y}\right)=\int_{-\infty}^{\infty} \int_{-\infty}^{\infty} \int_{-\infty}^{\infty} u(t, x, y) W^{*}(t, x-\alpha, y-\beta) e^{-j\left(\omega t-k_{x} x-k_{y} y\right)} d t d x d y
$$

where $\alpha$ and $\beta$ is the space indexes in $x$ and $y$ dimension. $W(t, x, y)$ is the 3D window function. In our study, a Hanning function is used to construct the $3 \mathrm{D}$ window $W(t, x, y)$, giving:

$$
W(t, x, y)=\left\{\begin{array}{cc}
0.5\left[1-\cos \left(2 \pi \frac{\sqrt{x^{2}+y^{2}}}{D_{r}}\right)\right] & \text { if } \sqrt{x^{2}+y^{2}} \leq D_{r} / 2 \\
0 & \text { otherwise }
\end{array}\right.
$$

where $D_{r}$ is the window diameter in space domain. The Fourier transform of the windowed portion will yield a frequency-wavenumber spectrum which only contains the local information of the windowed region around the spatial location $(\alpha, \beta)$. The result $L\left(\alpha, \beta, \omega, k_{x}, k_{y}\right)$ contains the local frequency-wavenumber spectra at different spatial locations. The local effective wavenumber distribution $k(\alpha, \beta)$, at the location $(\alpha, \beta)$ and interested frequency $\omega_{0}$ is then calculated via a weighted sum of the spectrum $L\left(\alpha, \beta, \omega_{0}, k_{x}, k_{y}\right)$,

$$
k(\alpha, \beta)=\frac{\sum_{\mathbf{k}} L\left(\alpha, \beta, \omega_{0}, k_{x}, k_{y}\right)|\mathbf{k}|}{\sum_{\mathbf{k}} L\left(\alpha, \beta, \omega_{0}, k_{x}, k_{y}\right)}
$$

The wavenumber distribution $k(\alpha, \beta)$ indicates how the wavenumber varies in the $2 \mathrm{D}$ space domain.

\section{WAVEFIELD SIMULATION AND MEASUREMENT}

\subsection{EFIT simulation}

3D EFIT simulations have been implemented in this study to aid in the acquisition of 3D wave propagation data. EFIT is a numerical method that is similar to staggered grid finite difference methods [26]. During the decades since finite integration technique was first applied to elastodynamics, many authors have reported using EFIT to investigate ultrasonic damage detection applications [27-29]. Previous work by Leckey et. al has shown that 3D ultrasonic EFIT 
simulations aid in understanding unexpected features in experimental Lamb wave data and that wavenumber domain analysis of simulation data can provide insight into mode conversion as Lamb waves interact with damage [21].

The EFIT code that we have implemented is parallelized using Message Passing Interface (MPI) and can be run efficiently on computing clusters. The simulations discussed in this work were run on NASA Langley Research Center's computing cluster (the "k-cluster"), which contains 252 dual socket quad core $2.66 \mathrm{GHz}$ Intel 5355 nodes We will not list the discretized EFIT velocity and stress equations or the spatial and temporal step size limitations that result from frequency considerations and stability conditions, since the equations are fairly lengthy and can be found in several references, such as [21,30]. For all simulations discussed in this work we used a spatial step size that meets the $\Delta x \leq \lambda_{\min }$ $/ 10$ requirement by setting $\Delta x=\lambda_{\min } / 36$ (where $\lambda_{\min }=c_{\min }$ /frequency, and $\mathrm{c}_{\min }$ was found using the dispersion curves).

The Lamb wave propagation in a large 2024 aluminum alloy plate of the dimensions $610 \mathrm{~mm} \times 510 \mathrm{~mm} \times 1 \mathrm{~mm}$ (material properties are listed in Table 1) was simulated by using EFIT. The schematic of the plate is shown in Figure 5a. In order to closely approximate the round shape $\mathrm{PZT}^{\dagger}(7 \mathrm{~mm}$ in diameter and $0.2 \mathrm{~mm}$ thick) actuation, the EFIT source excitation was implemented as an in-plane displacement occurring at the edges of the PZT sensor (at the location ). The excitation signal is a Hanning window smoothed three count toneburst. The excitation center frequency was chosen to be $360 \mathrm{kHz}$, where both the fundamental $\mathrm{S}_{0}$ and $\mathrm{A}_{0}$ Lamb wave modes are actuated. Additionally, we point out that the 3D EFIT simulation code is in Cartesian coordinates, and therefore tracks $v_{x}, v_{y}$, and $v_{z}$ velocity components. At the time $t=70.2 \mu s, v_{x}, v_{y}$, and $v_{z}$ velocity components from the EFIT simulation are shown in Figure 6.

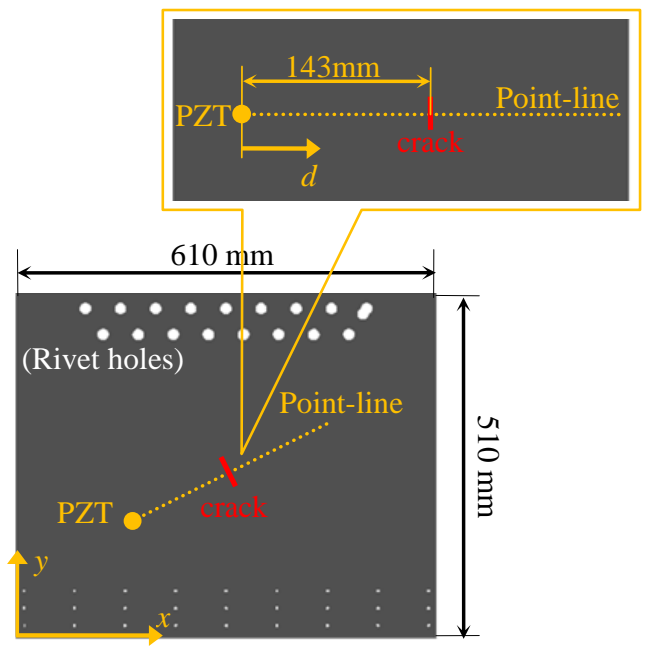

(a)

Figure 5 Lamb wave propagation in an aluminum plate: (a) schematic of the test specimen (not proportional, illustration purpose only); (b) SLDV experimental setup.

\subsection{Experimental verification}

To verify the EFIT simulations, the wavefield in the aluminum plate is also measured and visualized using a 1D SLDV [8-20] (model Polytec PSV-400-M2). This SLDV can measure the wave velocity/displacement component in the direction of the laser beam, based on the Doppler Effect on light waves. In this study, the specimen is vertically placed such that the laser beam from the 1D SLDV is normal to its surface and the out-of-plane velocity $v_{z}$ along a predefined grid is measured. The experimental measurement $v_{z}$ component is then compared to the EFIT simulation $v_{z}$ output. The overall test setup is shown in Figure $5 \mathrm{~b}$. The interrogation signal (360 kHz 3-count toneburst) is generated by an arbitrary waveform generator (Hewlett Packard 33120A) and then sends to the PZT actuator through a voltage amplifier. The out-of-plane response $v_{z}$ is then obtained by the 1D SLDV. The reflective tape is adhered to the plate surface to enhance the surface condition. 550 scan points along the scan line (in Figure 5a) are taken with a spatial resolution of $0.48 \mathrm{~mm}$ and a time sampling rate of $10.24 \mathrm{MHz}$. The measurement at each scan point is averaged 30 times to improve the signal quality.

\footnotetext{
${ }^{\dagger}$ APC851 http://www.americanpiezo.com/apc-materials/choosing.html
}

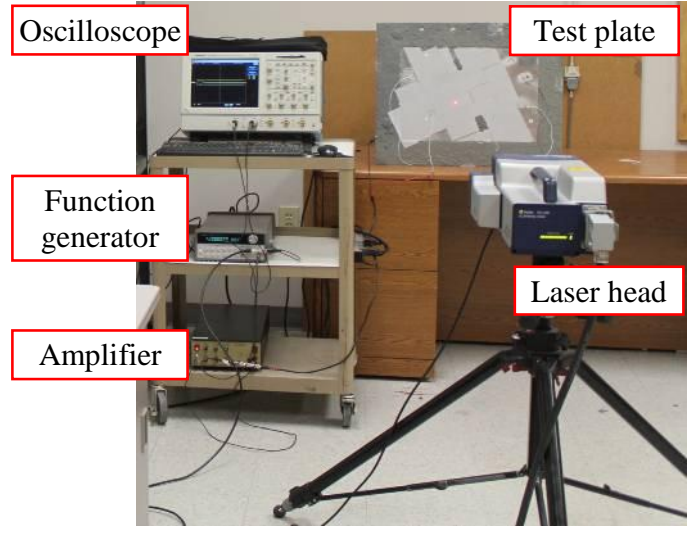

(b) 
The wavefield images of the out-of-plane velocity components from EFIT simulation and SLDV measurement are compared in Figure 7. Their wavefields appear similar in the two images. Comparisons of various wave representations have also been made for waveform, time-space, frequency-wavenumber representations, respectively. Waveforms extracted at the point $100 \mathrm{~mm}$ to the actuator are compared in Figure 8. The time-space wavefields along the selected line from EFIT simulation and SLDV measurement are compared in Figure 9. The simulation and experimental results match very well, showing a faster $S_{0}$ mode and a slow $A_{0}$ mode. Figure 10 compares their frequency-wavenumber representations. Both simulation and experimental results match well with the theoretical dispersion curves. Note that the amplitude of the $A_{0}$ mode is much higher than that of the $S_{0}$ mode due to the fact that the $A_{0}$ mode is out-of-plane motion dominated while the $S_{0}$ mode is in-plane motion dominated.
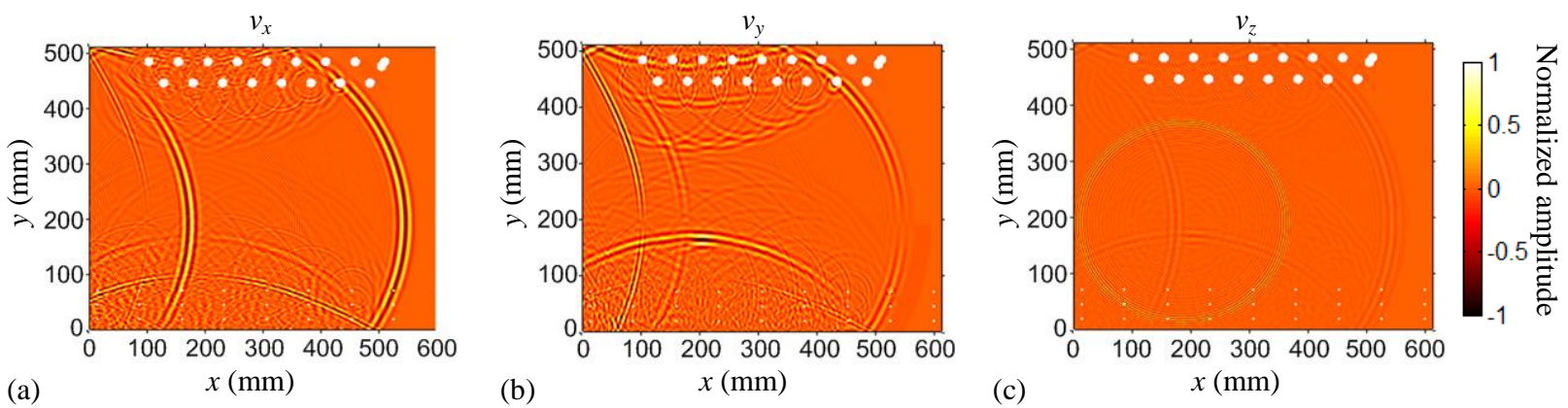

Figure $62 \mathrm{D}$ slices from the 3D EFIT simulation at the time $\mathrm{t}=70.2 \mu \mathrm{s}$ after the initial excitation: (a) $v_{x}$; (b) $v_{y}$; (c) $v_{z}$.
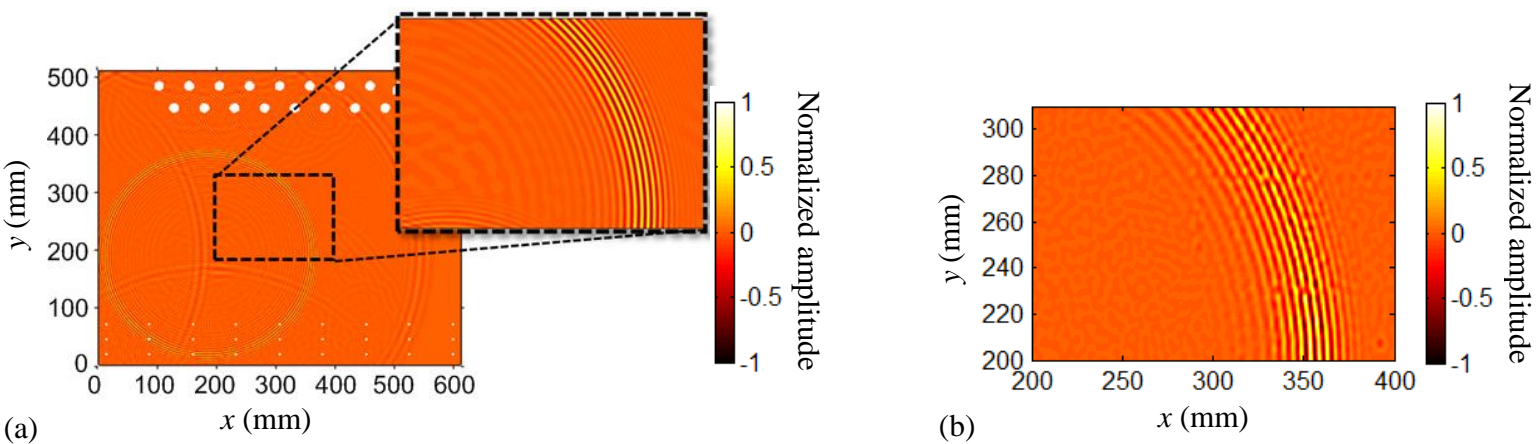

Figure 7 Comparison of the wavefield images ( $v_{z}$ out-of-plane components) at $70.2 \mu \mathrm{s}$ : (a) EFIT simulation, (b) SLDV measurement.

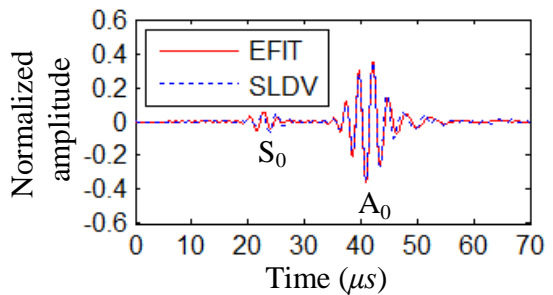

Figure 8 Comparison of the waveforms ( $v_{z}$ out-of-plane components) from EFIT simulation and SLDV measurement at the point $100 \mathrm{~mm}$ to the actuator. 

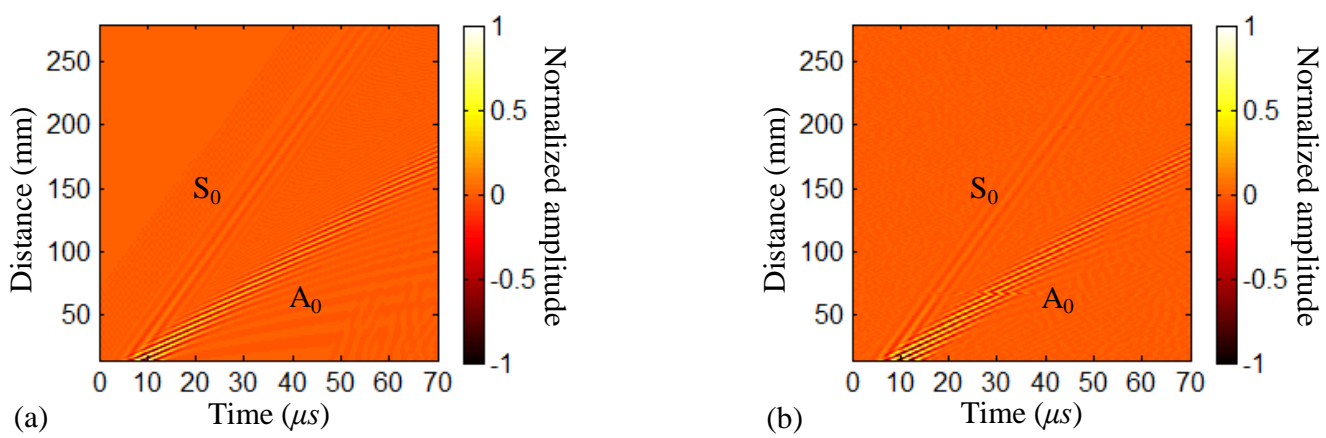

Figure 9 Comparison of time-space representations ( $v_{z}$ out-of-plane components): (a) EFIT simulation; (b) SLDV measurement.
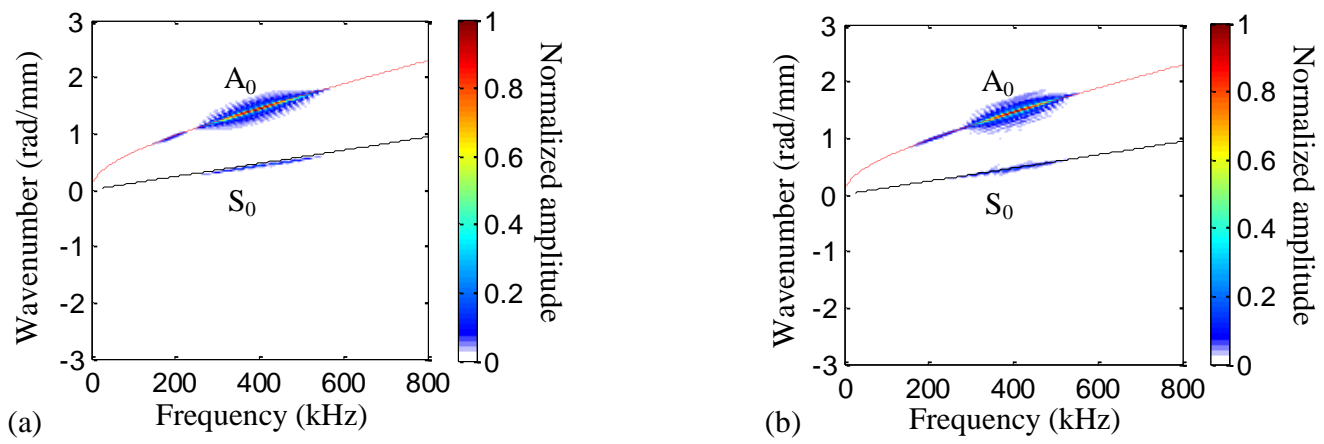

Figure 10 Comparison of frequency-wavenumber analysis results for pristine plate: (a) EFIT simulation; (b) SLDV experiment. Theoretical dispersion curves are also shown where $\mathrm{A}_{0}$ is the red dashed line and $\mathrm{S}_{0}$ is the black solid line.

\section{CRACK DETECTION}

The comparisons in section 3 show that EFIT and SLDV out-of-plane velocity $\left(v_{z}\right)$ components are in good agreement with each other. Since the in-plane and out-of-plane velocity components in the EFIT equations are not independent (they are coupled to each other via the stress equations), it is expected that in-plane EFIT data will also accurately represent Lamb waves in the plate. Hence, both in-plane $\left(v_{x}\right.$ and $\left.v_{y}\right)$ and out-of-plane $\left(v_{z}\right)$ components from the 3D EFIT simulation can be used to investigate how Lamb waves interact with the crack.

A $31 \mathrm{~mm}$ through-thickness crack centered at $(315 \mathrm{~mm}, 249 \mathrm{~mm})$ was included in the EFIT model by using stress-free boundary conditions [21]. The schematic of the cracked plate is shown in Figure 5a. The wavenumber analysis techniques described in section 2 are then applied to the 3D EFIT simulation data for crack detection.

\subsection{Frequency-wavenumber analysis}

The EFIT simulation results along the point-line which vertically pass through the crack (as shown in Figure 5a) are extracted for further analysis. The extracted time-space wavefield $u(t, d)$ can be plotted as amplitude (in color) versus time $t$ and spatial distance $d$ to the actuator. Figure 11a, b and c show the extracted time-space wavefields of $v_{x}, v_{y}$ and $v_{z}$ components for the pristine plate, respectively. After using time-space FT, the corresponding frequency-wavenumber spectra are plotted in Figure 11d, e and f, respectively. It is noted that the $\mathrm{S}_{0}$ mode has stronger in-plane components $\left(v_{x}\right.$ and $v_{y}$ ) while the $\mathrm{A}_{0}$ mode has a stronger out-of-plane component $\left(v_{z}\right)$. All frequency-wavenumber spectra show positive wavenumber components for both wave modes, indicating that they travel in the forward direction (i.e., no reflections occur for the pristine plate within the time length shown). 

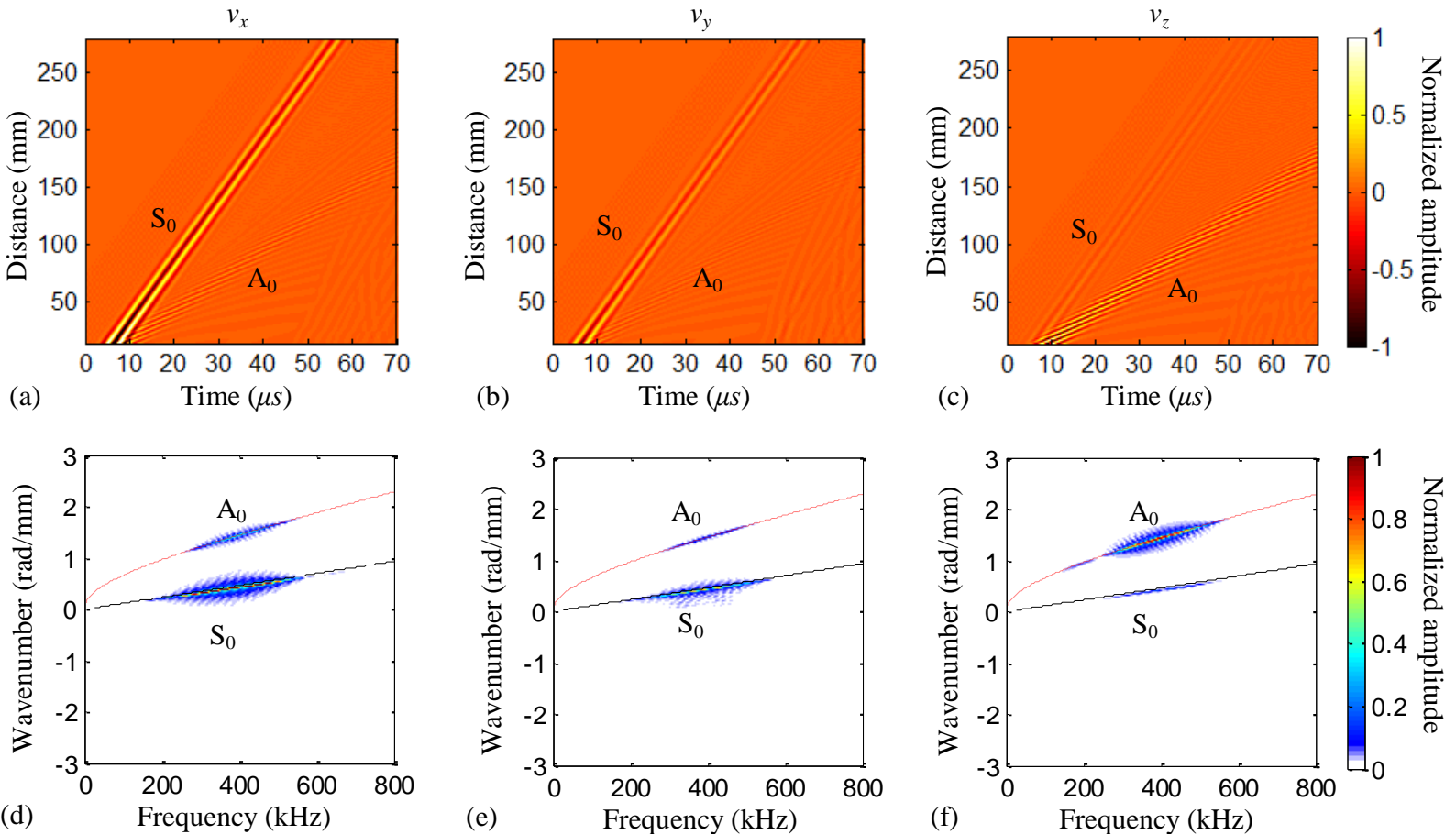

Figure 11 Frequency wavenumber analysis of 3D EFIT simulate data for the pristine plate: (a) time-space wavefield for $v_{x}$ component; (b) time-space wavefield for $v_{y}$ component; (c) time-space wavefield for $v_{z}$ component; (d) frequencywavenumber spectrum for $v_{x}$ component; (e) frequency-wavenumber spectrum for $v_{y}$ component; (f) frequency-wavenumber spectrum for $v_{\mathrm{z}}$ component.
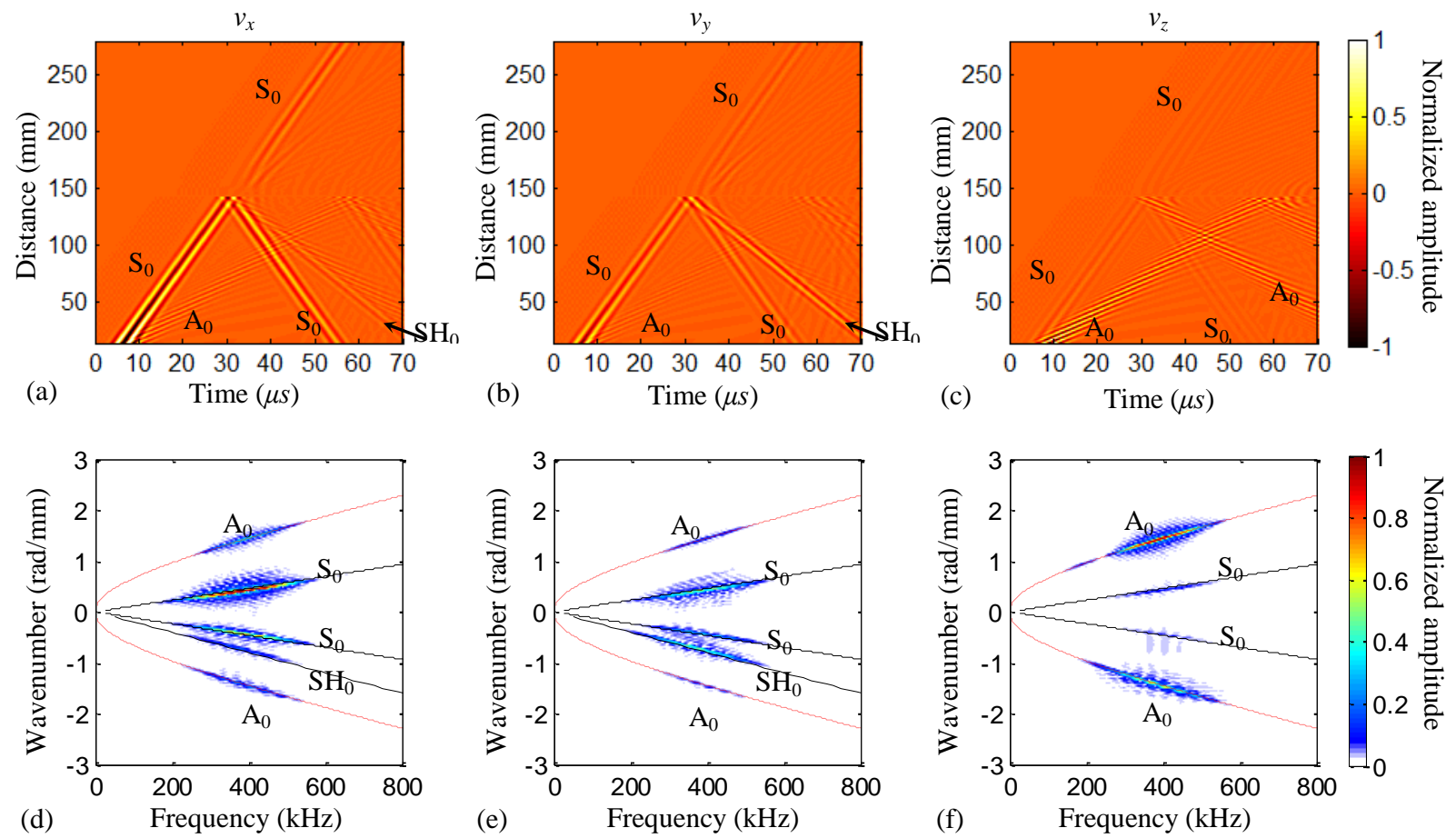

Figure 12 Frequency-wavenumber analysis of 3D EFIT simulate data for the cracked plate: (a) time-space wavefield for $v_{x}$ component; (b) time-space wavefield for $v_{y}$ component; (c) time-space wavefield for $v_{z}$ component; (d) frequency- 
wavenumber spectrum for $v_{x}$ component; (e) frequency-wavenumber spectrum for $v_{y}$ component; (f) frequency-wavenumber spectrum for $v_{z}$ component.

Figure 12 shows the time-space wavefields of $v_{x}, v_{y}$ and $v_{z}$ components for the plate with a crack, and corresponding frequency-wavenumber spectra, respectively. Comparing to the pristine results given in Figure 11, the wave propagation becomes very complicated after the modes interact with the crack damage. Figure 12 shows wave reflection, transmission, and mode conversion. For the in-plane components, both $\mathrm{A}_{0}$ and $\mathrm{S}_{0}$ are reflected by the crack, and part of the incident waves are transmitted through the crack. Meanwhile, the $\mathrm{SH}_{0}$ mode is created due to mode conversion occurring at the structural discontinuity (crack) from the incident $\mathrm{S}_{0}$ mode. For the out-of-plane component, the incident $\mathrm{S}_{0}$ mode is converted to an $\mathrm{A}_{0}$ mode at the discontinuity. Note that in the frequency-wavenumber spectra, negative wavenumbers represent waves that propagate opposite to the incident wave. All existing modes are highlighted in the frequency-wavenumber spectra, clearly discernible from each other.

\subsection{Space-frequency-wavenumber analysis}

Recall the time-space wavefield $u(t, d)$ along the point-line shown in Figure 5a. By using the short space 2D FT, spacefrequency-wavenumber representations can be obtained. To visualize the four dimensional data (where amplitude is the fourth dimension besides space, frequency, and wavenumber dimensions), space-wavenumber slides at a selected frequency are plotted, showing how wavenumber changes along propagation distance $d$ to the actuator. Figure 13 shows the space-wavenumber spectra of the pristine plate at the excitation frequency $360 \mathrm{kHz}$. For the pristine plate, incident $\mathrm{A}_{0}$ and $\mathrm{S}_{0}$ modes are present, and distributed around the wavenumber $1.39 \mathrm{rad} / \mathrm{mm} \mathrm{for} \mathrm{the} \mathrm{A}_{0}$ mode and 0.4 $\mathrm{rad} / \mathrm{mm}$ for the $S_{0}$ mode. These wavenumbers are consistent with theoretical $A_{0}$ and $S_{0}$ modes wavenumber values at 360 $\mathrm{kHz}$ (see Figure 1).

Figure 14 shows the space-wavenumber spectra for the cracked plate at the excitation frequency $360 \mathrm{kHz}$. In the spectra, reflected, transmitted and converted wave modes are present in addition to the incident waves. For the out-of-plane component $\left(v_{z}\right)$, both $\mathrm{A}_{0}$ and $\mathrm{S}_{0}$ are reflected. For the in-plane components $\left(v_{x}\right.$ and $\left.v_{y}\right)$, besides wave reflection, mode conversion also occurs. A converted $\mathrm{SH}_{0}$ mode is created and now present in both transmitted and reflected waves. The results show that both in-plane and out-of-plane components are important in the wave propagation analysis In all spectra, we can clearly see that the wave reflection and mode conversion occurs about the location $x=143 \mathrm{~mm}$, which is the crack location. Hence, the space-wavenumber analysis can be used for crack detection.

\subsection{Local wavenumber domain analysis}

For the study of the time-space wavefield $u(t, x, y)$, local wavenumber domain analysis was used [25]. Local wavenumber domain analysis is based on the assumption that presence of defects will significantly alter the dominant wavenumber in the local region of the defect. The analysis procedure involves windowing a local spatial region of the wavefield, applying the 3D FT, and calculating a weighted average of the wavenumber spectrum at the interested frequency of the incident wave. In this case, the Hanning window was used with the size approximately equal to twice the dominant wavelength in the wavefield ( $\lambda=3 \mathrm{~mm}$ for mode $\mathrm{A}_{0}$ mode in the out-of-plane wavefield and $\lambda=15 \mathrm{~mm}$ for mode $S_{0}$ in the in-plane wavefield). The out-of-plane results are presented in Figure 15a and show excellent agreement with the size of the crack, indicated by solid black lines. The increased indication at the crack is caused by the discontinuity of the wavefield created by the crack. The in-plane results are presented in Figure $15 \mathrm{~b}$ and show increased indication on the far side of the crack from the source. This is caused by both discontinuity in the wavefield and the presence of forward scattered $\mathrm{SH}_{0}$ waves.

\section{CONCLUSIONS}

This paper presented wavenumber analysis methods that can be used to study Lamb wave propagation. We expanded the wavefield analysis techniques presented in [14-16] to 3D full component wavefield data. The work presented here shows the importance of studying full 3D component wavefield data. Three wavenumber methods were presented: (i) frequency-wavenumber analysis via time-space FT, (ii) space-frequency-wavenumber analysis via a short space FT, and (iii) local wavenumber domain analysis via 3D windowed FT. In our study, the full 3D wavefield data (in-plane and outof-plane components) were obtained through 3D elastodynamic finite integration technique. The out-of-plane EFIT component was experimentally verified through comparisons to SLDV data. Both the time-space and frequencywavenummer representations of the two sets of data match very well. 
The application of wavenumber analysis for damage detection was demonstrated through the data analysis on a simulation example of an aluminum plate with a crack. Both in-plane and out-of-plane components were analyzed. The frequency-wavenumber analysis reveals all modes that exist in the wave propagation, including mode converted waves created due to interaction with the structural discontinuity. However, this method does not provide information regarding the location where the interaction occurs. With both short space frequency-wavenumber analysis and local wavenumber analysis, the spatial information is retained (1D and 2D, respectively). The space-frequency-wavenumber spectra at a selected frequency gives clear indication of where the wave reflection and mode conversion occur, i.e., where the structural discontinuity is located as a means for damage detection. In addition, the results show that both in-plane and out-of-plane information are important in the wave propagation and damage detection analysis. In the case presented in the paper, mode conversion from $\mathrm{S}_{0}$ mode to $\mathrm{SH}_{0}$ mode is only visible in the in-plane components. For crack detection, the short space frequency-wavenumber analysis has proven as an effective tool for crack detection while the local wavenumber analysis is shown to be useful for $2 \mathrm{D}$ crack sizing. The work also demonstrated that $3 \mathrm{D}$ simulation data can be utilized for Lamb wave damage detection investigations without costly experiment trials. In practice in a real-world setting, a sensor network could first be employed to locate a localized damage region; the SLDV and wavenumber analysis could then be used to narrow in on a precise crack location. Moreover, wavefield simulations combined with this type of analysis could help researchers predict what modes to expect to appear in experimental measurements (such as $1 \mathrm{D}$ or 3D SLDV).
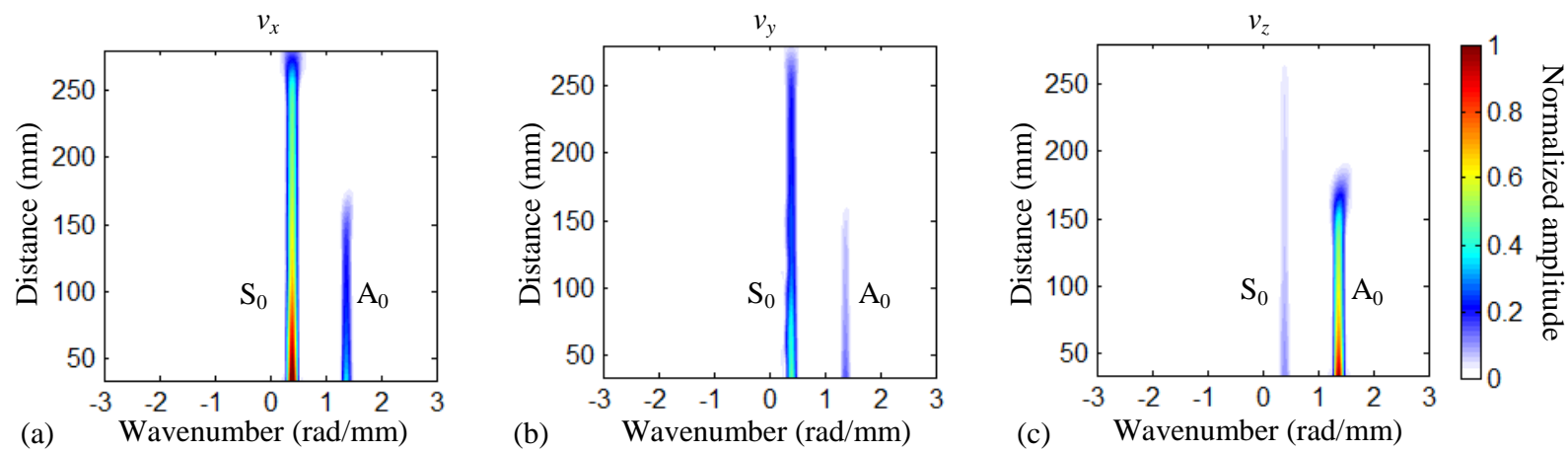

Figure 13 Space-frequency-wavenumber analysis of 3D EFIT data for pristine plate: (a) space-wavenumber spectrum at $360 \mathrm{kHz}$ for $v_{x}$ component; (b) space-wavenumber spectrum at $360 \mathrm{kHz}$ for $v_{z}$ component; (c) space-wavenumber spectrum at $360 \mathrm{kHz}$ for $v_{y}$ component.
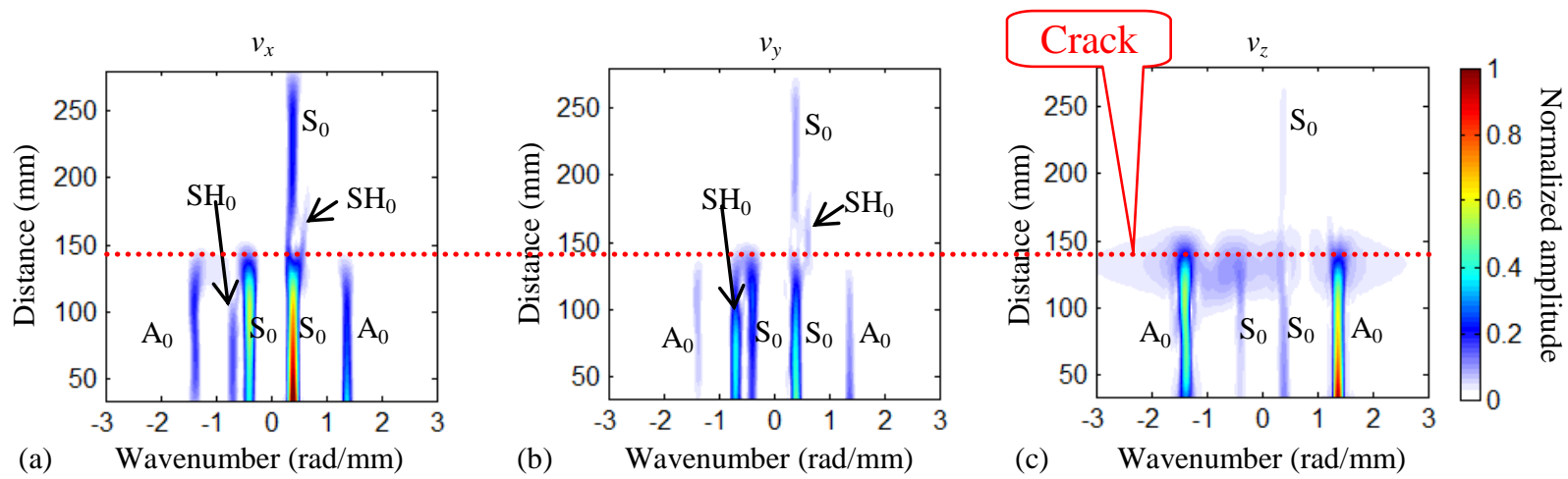

Figure 14 Space-frequency-wavenumber analysis of 3D EFIT data for the cracked plate: (a) space-wavenumber spectrum at $360 \mathrm{kHz}$ for $v_{x}$ component; (b) space-wavenumber spectrum at $360 \mathrm{kHz}$ for $v_{y}$ component; (c) space-wavenumber spectrum at $360 \mathrm{kHz}$ for $v_{z}$ component. 

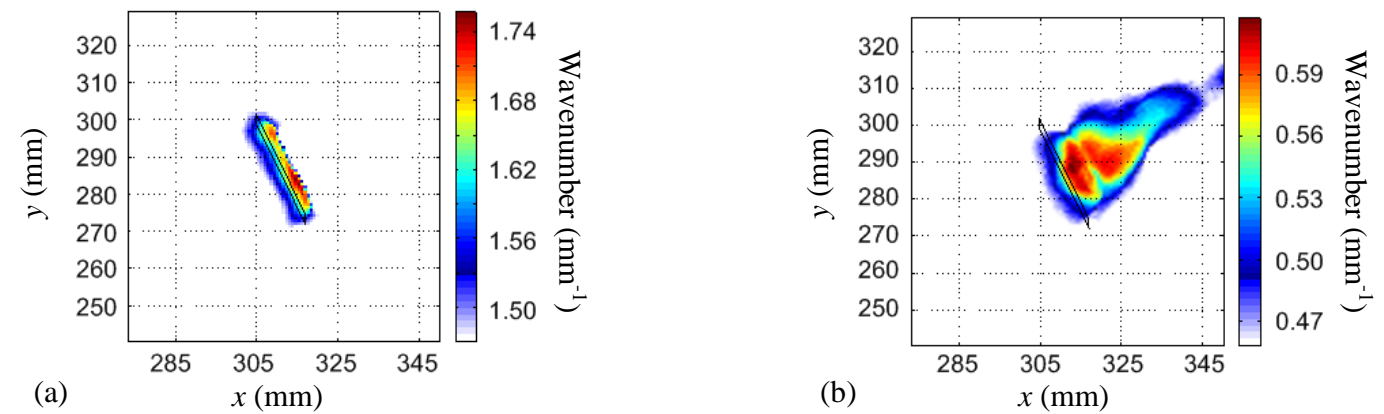

Figure 15 Local wavenumber domain analysis of 3D EFIT simulate data for the cracked plate: (a) out-of-plane (v $v_{x}$ component) result; (b) in-plane result (sum of the spectra for $v_{x}$ and $v_{y}$ component ).

\section{ACKONWLEDGEMENT}

Part of this work is conducted through the non-reimbursement space act umbrella agreement SAA1-1181 between South Carolina Research Foundation (SCRF) and the National Aeronautics and Space Administration (NASA) Langley Research Center. Part of this work is supported by the U.S. Nuclear Regulatory Commission award NRC-04-10-155 with program managers Mr. Bruce Lin and previously Mr. Gary Wang.

\section{REFERENCES}

[1] H. Lamb, "On waves in an elastic plate," Proceedings of Royal Society, A: Mathematical, Physical and Engineering Sciences, 93, 114-128 (1917).

[2] J. L. Rose, [Ultrasonic Waves in Solid Media] Cambridge University Press, (1999).

[3] D. C. Worlton, "Ultrasonic Testing with Lamb Waves," Nondestructive Testing, 15, 218-222 (1957).

[4] D. N. Alleyne, and P. Cawley, "The Interaction of Lamb Waves with Defects," Ieee Transactions on Ultrasonics Ferroelectrics and Frequency Control, 39, 381-397 (1992).

[5] R. P. Dalton, P. Cawley, and M. J. S. Lowe, "The Potential of Guided Waves for Monitoring Large Areas of Metallic Aircraft Fuselage Structure," Journal of Nondestructive Evaluation, 20(1), 29-46 (2001).

[6] W. H. Ong, and W. K. Chiu, "Redirection of Lamb Waves for Structural Health Monitoring," Smart Materials Research, 2012, 1-9 (2012).

[7] V. Giurgiutiu, [Structural Health Monitoring with Piezoelectric Wafer Active Sensors] Academic Press, (2008).

[8] E. D. Swenson, H. Sohn, S. E. Olson et al., "A Comparison of 1D and 3D Laser Vibrometry Measurements of Lamb Waves," Proceedings of SPIE, 7650, 765003 (2010).

[9] D. A. Hutchins, and K. Lundgren, "A Laser Study of Transient Lamb Waves in Thin Materials," Journal of Acoustic Society of America, 85(4), 1441-1448 (1989).

[10] M. J. Sundaresan, P. F. Pai, A. Ghoshal et al., "Methods of Distributed Sensing for Health Monitoring of Composite Material Structures," Composites, 32, 1357-1374 (2001).

[11] M. Kehlenbach, B. Kohler, X. Cao et al., "Numerical and Experimental Investigation of Lamb Wave Interaction with Discontinuities," Proceedings of International Workshop of Structural Health Monitoring, 421428 (2003).

[12] W. J. Staszewski, B. C. Lee, L. Mallet et al., "Structural Health Monitoring Using Scanning Laser Vibrometry: I. Lamb Wave Sensing," Smart Materials and Structures, 13, 1672-1679 (2004).

[13] B. Kohler, and J. L. Blackshire, "Laser Vibrometric Study of Plate Waves for Structural Health Monitoring (SHM)," Review of Progress in Quantitative Nondestructive Evaluation, 25, 1672-1679 (2006).

[14] M. Ruzzene, "Frequency-Wavenumber Domain Filtering for Improved Damage Visualization," Smart Materials and Structures, 16, 2116-2129 (2007).

[15]T. E. Michaels, M. Ruzzene, and J. E. Michaels, "Incident Wave Removal through Frequency-Wavenumber Filtering of Full Wavefield Data," Review of Progress in Quantitative Nondestructive Evaluation, 1096, 604-611 (2009). 
[16]T. E. Michaels, J. E. Michaels, and M. Ruzzene, "Frequency-Wavenumber Domain Analysis of Guided Wavefields," Ultrasonics, 51, 452-466 (2011).

[17] S. E. Olson, M. P. DeSimio, J. D. Matthew et al., "Computational Lamb Wave Model Validation Using 1D and 3D Laser Vibrometer Measurement," 7650, 76500M (2010).

[18] W. Ostachowicz, T. Wanddowski, and P. Malinowski, [Damage Detection Using Laser Vibrometry], (2010).

[19] H. Sohn, D. Dutta, J. Y. Yang et al., "Delamination Detection in Composites through Guided Wave Field Image Processing," Composites Science and Technology, 71, 1250-1256 (2011).

[20] M. D. Rogge, and P. H. Johnston, "Wavenumber imaging for damage detection and measurement," Review of Progress in Quantitative Nondestructive Evaluation, 31, 761-768 (2012).

[21]C. Leckey, M. D. Rogge, C. Miller et al., "Multiple-mode lamb wave scattering simulations using 3D Elastodynamic Finite Integration Technique," Ultrasonics, 52, 193-207 (2012).

[22] L. Yu, and C. Leckey, "Lamb Wave Based Quantitative Crack Detection using a Focusing Array Algorithm," Journal of Intelligent Material Systems and Structures, (2013).

[23] D. H. Johnson, and D. D. E., [Array Signal Processing: Concepts and Techniques] Prentice-Hall Inc., (1993).

[24] L. Cohen, [Time Frequency Analysis: Theory and Applications] Prentice-Hall Inc., (1994).

[25] M. D. Rogge, and C. Leckey, "Characterization of impact damage in composite laminates using guided wavefield imaging and local wavenumber domain analysis," Ultrasonics, (in press).

[26] F. Schubert, A. Peiffer, and B. Kohler, "The elastodynamic finite integration technique for waves in cylindrical geometries," Journal of the Acoustical Society of America, 104, 2604-2614 (1998).

[27] P. Fellinger, and K. J. Langenberg, "Numerical techniques for elastic wave propagation and scattering," Proceedings of IUTAM Symposium, 81-86 (1990).

[28]F. Schubert, "Numerical time-domain modeling of linear and nonlinear ultrasonic wave propagation using finite integration techniques-theory and application," Ultrasonics, 42, 221-229 (2004).

[29] J. Bingham, and M. Hinders, "3D elastodynamic finite integration technique simulation of guided waves in extended built-up structures containing flaws," Journal of Computational Acoustics, 18, 1-28 (2010).

[30] P. Fellinger, R. Marklein, K. J. Langenberg et al., "Numerical modeling of elastic wave propagation and scattering with EFIT-elastodynamic finite integration technique," Wave Motion, 21, 47-66 (1995). 